\title{
El potencial de la percepción social aplicada al análisis de la vulnerabilidad en planificación urbana
}

Ana Ruiz. Universidad San Jorge, Zaragoza, Espańa.

RESUMEN | En la actualidad, es escaso el papel que tiene la percepción social del espacio urbano en los documentos que guían el adecuado desarrollo urbano integrado de las ciudades. Una mejor comprensión de la influencia de las relaciones sociales dentro de la ciudad permite aportar nuevo conocimiento a los mecanismos subyacentes de vulnerabilidad y exclusión social en el espacio urbano. Este trabajo se centra en estudiar la relación existente entre el enfoque cuantitativo propio de los actuales informes de vulnerabilidad urbana y un enfoque cualitativo basado en la percepción social, desarrollado en el marco del proyecto de investigación Mapa de Riesgo Social de Zaragoza (España) durante los años 2014 a 2017. El análisis de la cartografía generada demuestra que el riesgo de aislamiento social no es mayor inequívocamente en las áreas actualmente caracterizadas como vulnerables, y revela la importancia que adquiere este tipo de estudios en la planificación urbana.

PALABRAS CLAVE | vulnerabilidad, planificación urbana, integración social.

ABSTRACT | Today, the role of the social perception of urban space is scarce in the documents that guide the adequate integration of the urban development of cities. A better understanding of the influence of social relationships within the city allows new knowledge to be brought to the underlying mechanisms of vulnerability and social exclusion in the urban space. This work focuses on studying the relationship between the quantitative approach of current urban vulnerability reports and a qualitative approach based on social perception, developed within the framework of the Social Risk Map of Zaragoza (Spain) research project between 2014-2017. The cartographical analysis shows that the risk of social isolation is not greater unequivocally in the areas currently characterized as vulnerable and reveals the importance of this type of research for city and urban planning activities.

KEYWORDs | vulnerability, urban planning, social integration. 


\section{Introducción}

Los primeros estudios centrados en la valoración del grado de vulnerabilidad y riesgo de exclusión refieren a la década de los años setenta. Coincidentes con la sistematización de la información censal, se enfocaron básicamente en conocer, con cierto grado de generalización, la estructura y funcionamiento de la sociedad. Para ello se definía un sistema de indicadores de carácter social (De Miguel, 1970, pp. 217-248; De Miguel, Díez \& Medina, 1967, p. 11), que incorporaba variables referidas a aspectos de pobreza material, nivel de rentas o distribución de bienes sociales escasos o de desigual distribución en la unidad vecinal, constituyéndose así en una de las primeras referencias del empleo del análisis sociológico en la planificación en España. Esta aproximación continuó prácticamente inalterada hasta una década más tarde, cuando desde el marco común europeo se estableció como línea prioritaria de trabajo el diseńo de medidas y políticas para la integración de los barrios vulnerables.

El estudio específico de los barrios vulnerables por parte de la disciplina urbanística distingue, por un lado, el escenario habitual de áreas donde se concentran focos de pobreza; y por otro, el actual problema multidimensional de zonas urbanas deprimidas como producto de complejas interacciones de factores económicos, sociales y espaciales. En términos generales, se argumenta que el problema de las zonas vulnerables no es la pobreza como tal, sino la existencia de numerosos casos en que "muchas personas pobres viven fuera de las zonas urbanas más deprimidas $\mathrm{y}$ muchas de las personas que viven en estas áreas no son pobres" (Organisation for Economic Co-operation and Development [OECD], 1998, p. 10). Se infiere que no son los bajos ingresos los que caracterizan a estos barrios, sino una combinación de circunstancias ambientales, sociales y económicas que estimulan la alienación y generación de situaciones de vulnerabilidad y exclusión social. En otras palabras, se comprueba que los entornos urbanos que no sufren las privaciones de las zonas más pobres no parecen inmunes a los problemas de exclusión social (Atkinson \& Kintrea, 2001; Atkinson, Cantillon, Marlier \& Nolan, 2002).

La definición metodológica de la vulnerabilidad social ha sido ampliamente estudiada (Alguacil, 2006; Arias, 2000; Keestelot, 2008) y apunta, en última instancia, a aquellos ámbitos espaciales cuyo desarrollo económico se ve restringido, la cohesión social debilitada y en los que se generan altos costes ambientales (OECD, 1998). Este fenómeno se ha caracterizado principalmente en términos de indicadores cuantitativos, que evalúan aspectos como la proporción de población joven con respecto al total, altas tasas de monoparentalidad, niveles de ingresos muy bajos y altos niveles de dependencia de transferencias de ingresos, bajos niveles de mezcla sociolaboral, altos niveles de actividad económica informal, o pocas empresas comerciales locales y acceso deficiente a centros minoristas, entre otros. La diferencia de estos estudios respecto de los anteriores es clave, puesto que el propósito de poner en medida el concepto de vulnerabilidad depende de otras variables necesarias de considerar. Uno de los principales giros frente a décadas pasadas corresponde a la consideración de variables que refieren al porcentaje de espacios libres sobre lo construido, a la calidad ambiental, las comunicaciones o al nivel de proximidad de los usos 
complementarios y asistenciales con respecto a los residenciales. Al utilizar el conjunto de datos estadísticos, los estudios han demostrado un problema multidimensional en la identificación de las áreas urbanas en dificultades (Álvarez \& Roch, 2010; Conway \& Konvitz, 2000). Esta conjunción de consideraciones de tipo físico, social y económico modifica la escala de medida hacia el valor del espacio urbano como ámbito de integración efectivo. Su aplicación práctica ha sido extensamente referida, siendo numerosos los estudios que lo ejemplifican. España cuenta con el Observatorio Nacional de Vulnerabilidad Urbana como proyecto desde el que se desarrollan estudios específicos, con objeto de identificar para el conjunto del país estas áreas desfavorecidas, basándose para ello en los censos de población y vivienda. Para el censo de 1991 se desarrolló una primera metodología, que tomaba como criterios las tasas de paro, de población analfabeta y sin estudios, de población en viviendas que no cuentan con aseo o baño y de inmigración extracomunitaria (Ministerio de Fomento, 1990). La metodología sería depurada para el censo de 2001 y su adenda de 2006, estableciendo una batería de indicadores divididos en cuatro secciones: vulnerabilidad sociodemográfica, vulnerabilidad socioeconómica, vulnerabilidad residencial y vulnerabilidad subjetiva (Hernández-Aja et al., 2013).

Algunos trabajos paralelos que, de un modo u otro, toman estos cuatro tipos de vulnerabilidades como base de su estudio incorporan una optimización y mejora metodológica asociadas a estas políticas de regeneración de barrios (Bruquetas, Moreno \& Walliser, 2005). Al tratar de caracterizar las áreas vulnerables del medio urbano, Temes (2014) refiere que su origen no ha de buscarse únicamente en la forma urbis, sino en la compleja y frágil red de vínculos y relaciones socioeconómicas que moldean realmente la ciudad, proponiendo una aproximación al problema a través de una metodología fundada en una selección de criterios cuantitativos estadísticos más elaborada que en casos anteriores, y centrada en la noción de exclusión social. Por su parte, Ruiz y Alfaro (2017) incorporan en su estudio la condición modificable de los diferentes factores que explican la diversidad económica, social, ambiental y cultural de la ciudad. La metodología de análisis que proponen permite detectar las áreas susceptibles de intervención que tienen mayor poder de transformación del tejido residencial a corto-medio plazo, como óptimas para ser objeto de intervención en términos de rehabilitación urbana. Este enfoque proporciona información precisa para inferir que aquellas zonas declaradas como áreas de regeneración y renovación urbana que quedan fuera de los sectores más modificables requerirán, además, otras acciones más a largo plazo (estructurales) o instrumentos para su mejora. Es decir, su estudio permite obtener información acerca de si la acción de regeneración urbana debería contar con otras acciones complementarias al empleo exclusivo de acciones a corto-medio plazo de carácter urbanístico-arquitectónico.

Ahora bien, si es cierto que una revisión pormenorizada de todos estos estudios nos brinda una rica muestra de las diferentes variables empleadas y los métodos de análisis estadístico a la hora de establecer los mecanismos de medida de la vulnerabilidad urbana, también es cierto que todos ellos mantienen una mirada del concepto de vulnerabilidad desde lo cuantitativo. Los estudios existentes no han examinado lo suficiente si la vida social cívica podría influir en la configuración de las zonas urbanas caracterizadas como vulnerables. El objetivo fundamental no es 
solo identificar estas áreas mediante un enfoque cuantitativo, sino descubrir cómo funcionan estas áreas desde el punto de vista social. Esta es una limitación crítica, porque no se ha demostrado que el riesgo de aislamiento social sea mayor en las áreas vulnerables identificadas.

Aunque de manera más limitada, sí se encuentran referencias de autores que, al hablar de vulnerabilidad y exclusión social desde la planificación, subrayan que estos términos no pueden considerarse como una definición cerrada, debiéndose tratar como un concepto cualitativo (Maclaren, 1996; Turcu, 2012). Esto supone que el nivel de vulnerabilidad presenta una relación de dependencia con respecto a las percepciones y relaciones de cotidianeidad existentes en cada una de las comunidades urbanas, o unidades de barrio. Por ende, si estas no se consideran en el proceso de diseño metodológico, los resultados obtenidos en un barrio pueden distar del nivel de vulnerabilidad real. Desde el trabajo social y la sociología, existen ciertas investigaciones que confieren importancia a la percepción social como enfoque cualitativo que puede ayudar a caracterizar espacios vulnerables. Raya $(2007,2010)$ aborda la construcción de herramientas para la medición de la exclusión social desde la perspectiva del trabajo. Otras investigaciones se centran en el papel de la brecha digital y la neogeografía en el establecimiento de redes de apoyo instantáneas (Murie \& Musterd, 2004) y no basadas en un espacio físico en continuidad (Haklay, 2013; Rana \& Joliveau, 2009). Todo lo anterior lleva a subrayar que una mejor comprensión de la influencia de las relaciones sociales dentro de la ciudad es importante, pues aporta nuevo conocimiento a los mecanismos subyacentes de vulnerabilidad y exclusión social en el espacio urbano.

\section{Mapas sociales y percepción social a nivel de barrio}

El estudio de la esfera social en la ciudad pone de manifiesto la importancia de las redes de apoyo mutuo (familiar, vecinos o minorías étnicas), al configurarse como recurso prioritario a la hora de hacer frente a los procesos de exclusión social. Se ha comprobado que una red de convivencia y apoyo social no solo garantiza cierto nivel de estabilidad familiar y comunitaria, sino que ese nivel primario de organización social (Cooley, 1909) se torna una herramienta útil a la hora de mantener ciertas condiciones de vida y de autonomía frente a situaciones de vulnerabilidad. Así lo sustentan trabajos como los de Elliott et al. (1996) y Kingston, Huizinga y Elliot (2009). El estudio de la red de apoyo social es fundamental en la investigación sobre la ciudad y la planificación urbana, si se pretende alcanzar de manera propia y durable en el tiempo unas condiciones de calidad de vida equivalentes para los distintos sectores sociales, con independencia de intervenciones o condicionantes externos y del lugar de residencia. Esta aproximación es especialmente importante en aquellos casos donde son menores tanto las oportunidades laborales como las prestaciones de bienestar social, ya que las redes de apoyo social se ven ya de por sí debilitadas en el escenario actual de reducción del tamaño de las familias, de individualización, de reducción de la dependencia mutua y en las esferas geográficamente expansivas de la vida cotidiana (León \& García-Carpintero, 2017). 
Según Musterd y Murie (1999), la posibilidad de evaluar la presencia de esta esfera social se centra en la cantidad de relaciones sociales que tiene una persona (red social), las funciones que cumplen las personas o grupos que configuran la red social de un individuo y los efectos que tienen en el bienestar individual (apoyo social), o las relaciones con funciones de apoyo en momentos críticos de necesidad y demanda de apoyo (red de apoyo). La ausencia de esta última, de la red de apoyo, supondría una señal de exclusión y aislamiento social. Por ello, su objetivo se concreta en distinguir los diferentes modos en los que las personas socialmente excluidas abordaban su situación, de acuerdo con la relación entre la exclusión social y el debilitamiento de las redes sociales o de solidaridad.

Los estudios han demostrado que la manera como se configura y percibe un espacio urbano constituye un factor básico a la hora de asegurar cierta intensidad de relaciones vecinales de cotidianeidad, o de posibilitar su debilitamiento, atendiendo a elementos tanto físicos como sociales (Appleyard \& Lintell, 1972; Gehl, 2010). Sin embargo, es importante subrayar que es más escaso el trabajo que examina, desde una perspectiva de sostenibilidad social, si las relaciones sociales de cotidianeidad pueden influir en el diseño de herramientas de planificación urbana. Esta es una cuestión esencial, puesto que la propia disciplina urbana carece de instrumentos suficientes a la hora de incorporar con garantías el ámbito de lo social en el desarrollo y diseño de la ciudad.

El proyecto Mapa de Riesgo Social se ha centrado precisamente en la necesidad de concretar la dimensión medible de estas redes de apoyo, no solo en su dimensión operativa sino también en la de las propias observaciones, es decir, la cartografía de las relaciones sociales que se producen (León \& García-Carpintero, 2017; RuizVarona \& León-Casero, 2017). El interés se concreta en evaluar y medir la intensidad de las redes de apoyo de manera tal que puedan llegar a inferirse los potenciales riesgos que afecten a un individuo en un contexto urbano determinado. Esta cuestión se aborda a través de un análisis cualitativo que investiga cómo operan, desde el punto de vista de la percepción social, las redes de apoyo identificadas en el conjunto de la ciudad o área urbana. De hecho, la percepción y caracterización de las áreas de riesgo social refuerzan el compromiso trazado en las actuales políticas en materia de vulnerabilidad urbana, en cuanto a diseñar los modelos de análisis y criterios que determinan la existencia de áreas susceptibles de rehabilitación urbana en la ciudad.

En términos generales, la posibilidad de analizar el entorno urbano desde una perspectiva convivencial se desarrolla -además de vía el análisis de información proveniente de los censos de vivienda y demografía- a través de la georreferenciación de información temática que ha sido recogida previa investigación sobre el terreno. Este enfoque supone presentar al ciudadano como elemento esencial de la ciudad al considerarlo fuente primaria de la investigación, de manera tal que se tenga en cuenta la propia percepción de quienes ocupan y habitan la ciudad en sus diversas manifestaciones, y no únicamente los registros del propio observador (García-Bueno, 2017). Junto con ello, los Sistemas de Información Geográfica (sIG) se convierten en una herramienta indispensable para visualizar la vida social de un 
entorno urbano concreto, puesto que contar con información georreferenciada es clave a la hora de elaborar una cartografía del riesgo social.

\section{Análisis de datos y métodos de investigación}

La investigación a través de mapas sociales cuenta con precedentes, si bien estos se centran fundamentalmente en el uso de técnicas de análisis cuantitativo multivariante aplicado a casos de estudio a escala urbana (Goicoechea, 2014; Torres, 1993) o territorial; este último, fundamentalmente en términos de riesgos sociales frente a desastres naturales (Cutter, Boruff \& Shirley, 2003; Lee, 2014). Sin embargo, son escasos aquellos que incorporan un enfoque cualitativo como medida del riesgo y caracterización de la vulnerabilidad urbana.

Un aspecto esencial para toda metodología de análisis consiste en la delimitación de sus unidades primarias de análisis (Alguacil, 2011; King, Keohane \& Verba, 2000). Frente a los estudios de vulnerabilidad urbana previos, de marcada metodología de análisis cuantitativo, el proyecto Mapa de Riesgo ha definido el barrio como unidad espacial de análisis. Es evidente que la posibilidad de realizar dicha delimitación en función de divisiones administrativas (juntas municipales, códigos postales, unidades de distrito o unidades censales) hubiera facilitado el análisis e interpretación de los datos estadísticos recopilados de diversas fuentes públicas. Sin embargo, se ha comprobado que es precisamente a nivel de barrio donde se producen las conexiones de dependencia y sentimiento de pertenencia espacial de un individuo con respecto a una colectividad (Campbell, Henly, Elliott \& Irwin, 2009; Coulton, Korbin, Chan \& Su, 2001; Guest \& Lee, 1984; Keller, 1968). Asimismo, es precisamente a esta escala de barrio donde, de manifestarse espacialmente los sentimientos vecinales de pertenencia desde un punto de vista social, se producen las relaciones vecinales de cotidianeidad que le permiten configurarse finalmente como la unidad espacial mínima donde se puede lograr la autosuficiencia para cubrir las necesidades de la vida cotidiana (Obst \& White, 2004). Esta autosuficiencia, o autonomía para la reproducción, no solo queda caracterizada por la facultad que tiene un entorno de abastecer a quienes lo habitan de productos y servicios necesarios para la vida cotidiana y para los que no se requiere un comercio o servicio especializado (García-Bueno, 2017). También se caracteriza por la identificación espacial de las relaciones sociales que subyacen a la estructura morfológica de la ciudad, explicadas en términos de generación de escenarios de cotidianeidad entre los vecinos (Lofland \& Lofland, 1995). Ambos niveles de autosuficiencia quedan complementados por la existencia de agrupaciones y asociaciones que, representando intereses colectivos, se encargan de generar o intensificar determinadas redes de convivencia positivas en un determinado espacio urbano comunitario (García-Bueno, 2017).

La superposición de estos niveles de autosuficiencia genera la delimitación de la unidad de convivencia y es motivo por el que la delimitación de barrios fijada inicialmente ha podido quedar sujeta a modificaciones; por ejemplo, expandiéndose en aquellos casos donde no existan servicios de proximidad, puesto que, en este supuesto, las personas deben ampliar su espacio de relaciones y actividades cotidianas a otras áreas que cuenten con tales servicios. El carácter dinámico inherente 
a la unidad de convivencia ha hecho prever, como uno de los resultados de la investigación, la identificación de alteraciones a la actual delimitación de unidades de barrio (figura 1).

FIGURA I | Delimitación de las unidades de barrio de Zaragoza

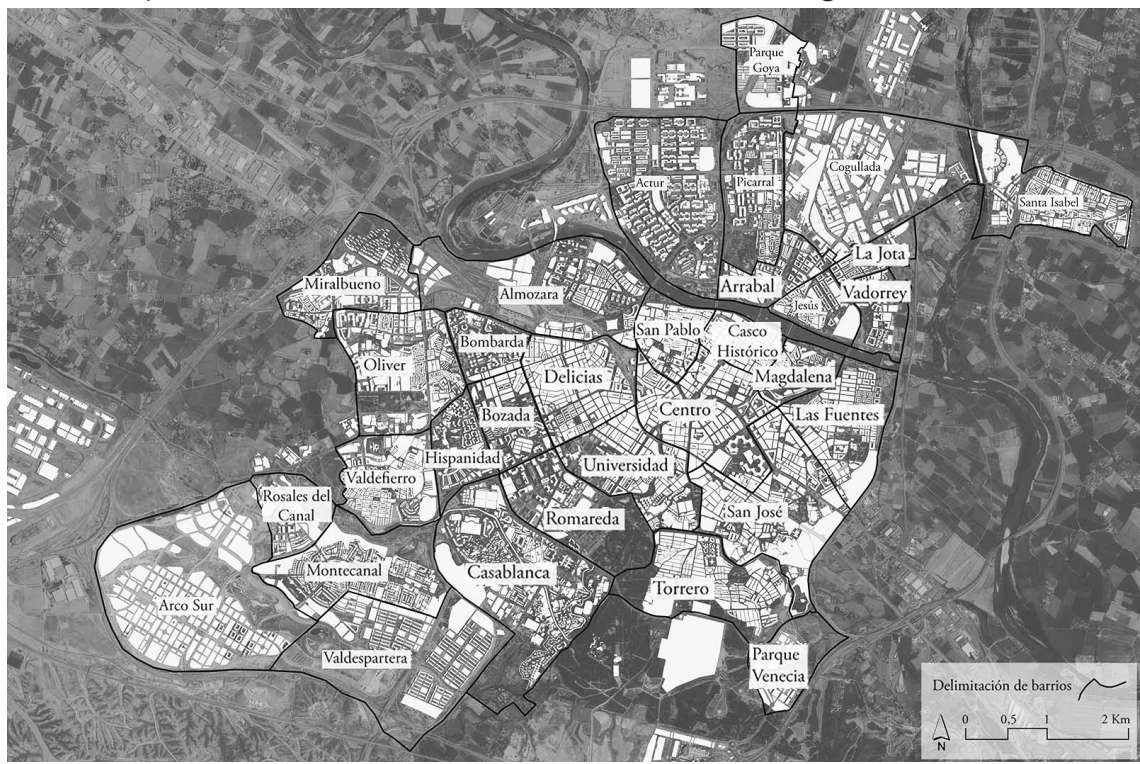

FUENTE ELABORACIÓN PROPIA, 20 I 7

Comparar, a través de un estudio morfológico del espacio urbano, los patrones de vulnerabilidad urbana actuales y los significados que los participantes atribuyen a las características y condiciones de convivencia a nivel de barrio $-\mathrm{y}$, por tanto, descubrir cómo funciona el barrio desde un punto de vista social-, permite evaluar la percepción social en su potencial de ofrecer explicaciones respecto de si se producen, y en qué medida, las situaciones de vulnerabilidad previstas y qué consecuencias sociales tienen. Para ello, el método de investigación incorpora técnicas de naturaleza cuantitativa como principal avance sobre los actuales mecanismos de evaluación del riesgo social y la vulnerabilidad urbanas.

\section{Mapa de diversidad urbana y espacio público}

En primer lugar se realiza una medición de los productos y servicios necesarios para la vida cotidiana que caracterizan los diferentes tejidos urbanos de cada una de las unidades de análisis. Para ello se realizó trabajo de campo durante 2014 a 2017, en el que se ha recogido información de cada uno de los usos de los locales en planta baja del conjunto de la ciudad de Zaragoza (Cámara, León \& Ruiz, 2016). La base espacial que se ha tomado como referencia es la proporcionada por la Dirección General del Catastro a través de su Sede Electrónica, ${ }^{1}$ correspondiente a la cartografía vectorial

1 Gobierno de España, Ministerio de Hacienda, Sede Electrónica del Catastro. http://www. sedecatastro.gob.es/ 
urbana de la ciudad, y complementada por los documentos referidos al Planeamiento General de la ciudad facilitado por el Ayuntamiento de Zaragoza, ${ }^{2}$ si bien se ha modificado en aquellos casos en que las dimensiones de los locales de planta baja tomadas in situ no se correspondieran con las proporcionadas por el Catastro.

\section{FIgURA 2 Patrón de distribución de usos generales en planta baja de Zaragoza}
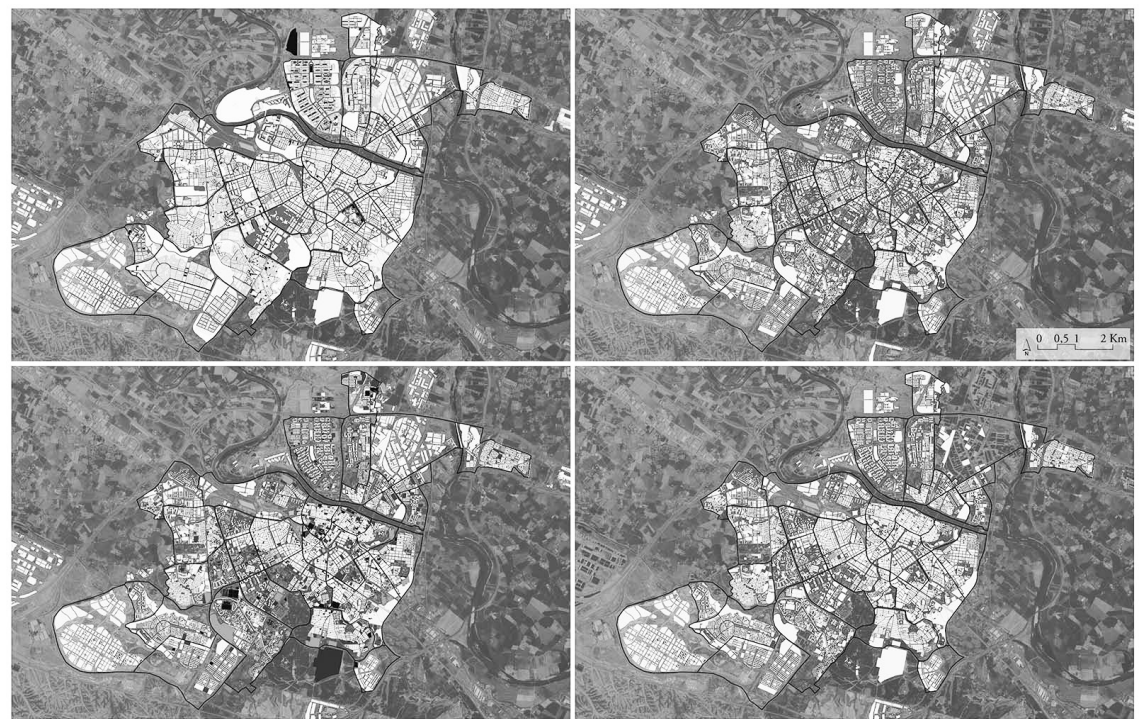

FUENTE ELABORACIÓN PROPIA, 2017

Conviene puntualizar que las geometrías de cada uno de los locales referidos en la cartografía elaborada no tienen carácter vinculante, puesto que en ocasiones ha sido difícil acceder a los mismos para comprobar los fondos o el uso de los patios de manzana, que, en general y salvo excepciones, han sido considerados como uso residencial colectivo. Por ello, el interés principal no reside tanto en términos numéricos, de manera que se establezcan comparativas de superficies de cada uso por habitante o por el conjunto de los usos, sino en términos cualitativos. Contar con información desagregada para cada barrio permite conocer el funcionamiento de la ciudad en estos últimos términos, por ejemplo, analizando el potencial de uso comercial a nivel de barrio con respecto al conjunto de la ciudad, la variabilidad y diversidad de usos o de concentración en torno a determinados elementos urbanos.

A este respecto, se ha definido un conjunto de cinco usos generales en los que agrupar cada una de las actividades de planta baja estudiadas. La figura 2 muestra una primera cartografía temática para el conjunto de la ciudad, ordenada según las categorías nominales de espacio libre (izquierda-superior), equipo urbano y servicios públicos (izquierda-inferior), uso terciario (derecha-superior) e industrial (derecha-inferior), en el que identificar el patrón de distribución de los diferentes usos agrupados.

2 Ayuntamiento de Zaragoza, Urbanismo/Memoria. https://www.zaragoza.es/ciudad/urbanismo/ planeamiento/pgouz/memoria.htm 
FIgura 3 | Patrón de distribución de usos específicos en planta baja de los barrios de La Almozara (superior) y Casco Histórico (inferior) en Zaragoza

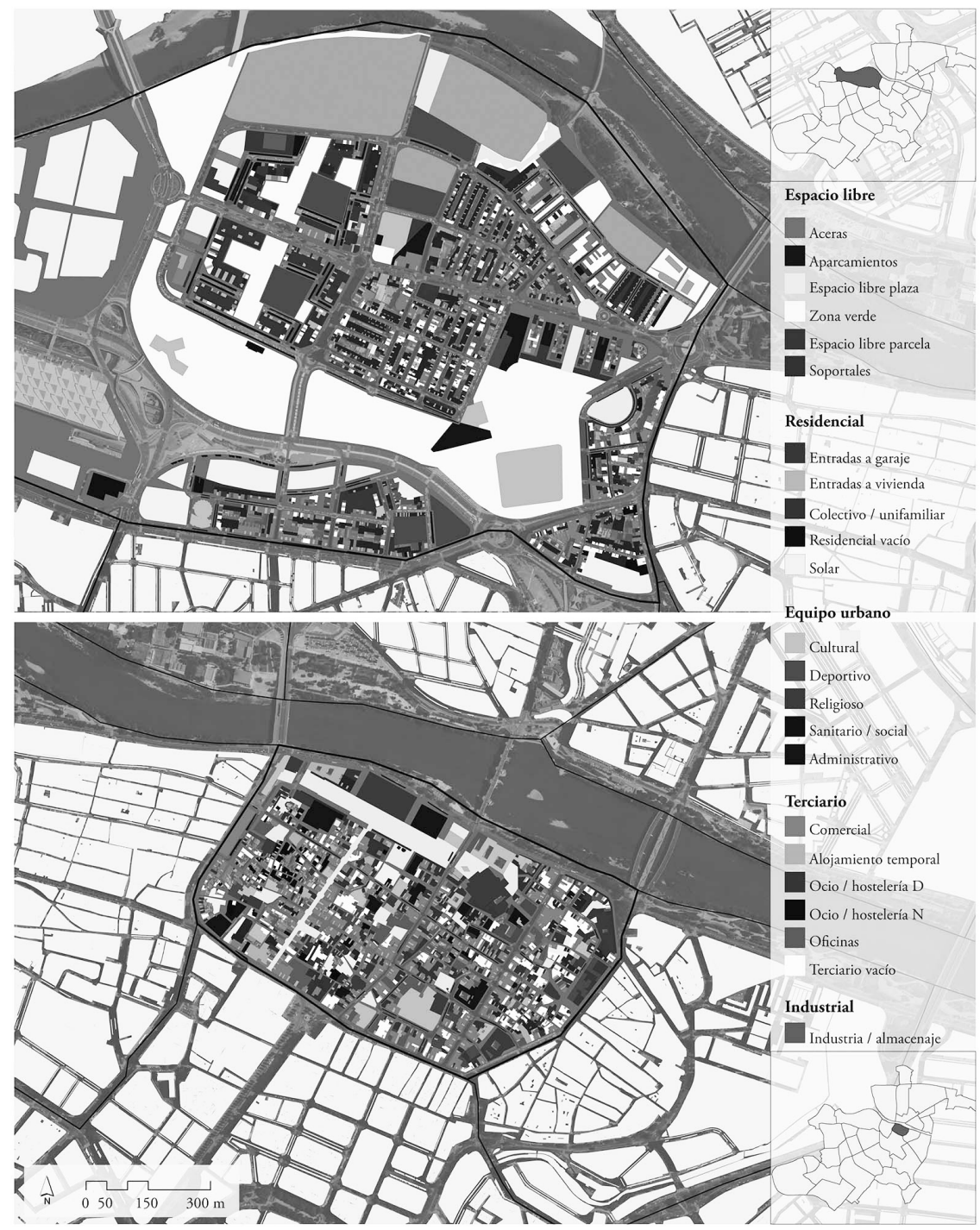

FUENTE ELABORACIÓN PROPIA, 2017

Cada una de estas categorías presenta niveles de desagregación mayores, según nivel de especialización y condición de uso en activo o cese de actividad. La figura 3 ofrece un mapa más en detalle, a nivel de barrio, en el que se compara los usos específicos en planta baja de dos barrios de la ciudad de Zaragoza ubicados ambos en su margen derecha, correspondientes a La Almozara y Casco Histórico, por 
considerarse contrastables no solo morfológicamente, sino en relación con el marco temporal en que se inscribe su desarrollo urbano y su condición de vulnerabilidad. ${ }^{3}$

\section{Mapa social y de percepción subjetiva}

En segundo lugar, se diseña el método para obtener información sobre las características y condiciones de convivencia a nivel de barrio para el conjunto de la ciudad de Zaragoza. Los principales contenidos por analizar a nivel de barrio como referencias clave para la valorización de la convivencia y de los riesgos en un entorno vecinal, han sido organizados, en términos generales, en cuatro categorías nominales: atractores, actividades vecinales espontáneas e institucionalizadas, espacios de riesgo por presencia marginal y conflictividad social y, por último, espacios deteriorados físicamente o con dificultades de acceso material. Mientras que el término de "atractor" hace referencia a aquellos elementos del espacio urbano que favorecen relaciones vecinales de cotidianeidad positivas, el de "espacio de riesgo" pretende reflejar aquellos que las debilitan (García-Bueno, 2017).

Esta información se obtiene a través de técnicas observacionales y conversacionales de investigación cualitativa; entre ellas, la observación presencial del espacio público según un guion de observación, y la realización de entrevistas en profundidad estructuradas a informantes clave. La guía de observación permite generar información homogénea para el conjunto de los barrios analizados acerca del uso de los espacios urbanos, incorporando especificidades como frecuencias, intensidades y perfil de población que lo frecuentan y otorgando especial importancia a la identificación de espacios de riesgo y deteriorados. Por su parte, las entrevistas a informantes clave permiten complementar el análisis de cada barrio con un aporte de información proporcionado por determinadas personas que gozan de una especial vinculación con el barrio (García-Bueno, 2017). Contar con una cartografía de los elementos que caracterizan el nivel de convivencia y riesgo a nivel de barrio es importante, puesto que de su estudio puede extraerse información precisa acerca no solo del patrón de distribución y conformación de la red de atractores, sino también de la influencia que ejercen las actividades vecinales (por ausencia o presencia) en el espacio urbano y en la aparición de áreas de riesgo y de atractores. Esta aproximación también permite plantear una gradación de tales redes de convivencia y apoyo social de cada uno de los barrios con respecto al resto. Las metodologías previas no tienen en consideración la red de apoyo social y, en general, queda asumido que un barrio caracterizado como vulnerable no va a contar con una valiosa red de apoyo social. Sin embargo, esto no se ha puesto en medida. Para comprobar hasta qué punto existe relación entre la condición de vulnerabilidad y los patrones de relación social en la ciudad, el trabajo no solo ha considerado los barrios caracterizados como vulnerables por el informe publicado por el Observatorio de Vulnerabilidad Urbana, sino que ha ampliado el estudio a otros barrios de la ciudad. Continuando con el ejemplo a escala de barrio anterior, la figura 4 refiere un ejemplo de cartografía social que se ha obtenido como resultado de aplicar esta metodología, en la 
que se distinguen los atractores, actividades vecinales (espontáneas e institucionalizadas), espacios de conflicto y deteriorados para los barrios de La Almozara y Casco Histórico.

FIgURA 4 Patrón de distribución de atractores, actividades vecinales y espacios de conflicto correspondientes a los barrios de La Almozara (superior) y Casco Histórico (inferior) en Zaragoza

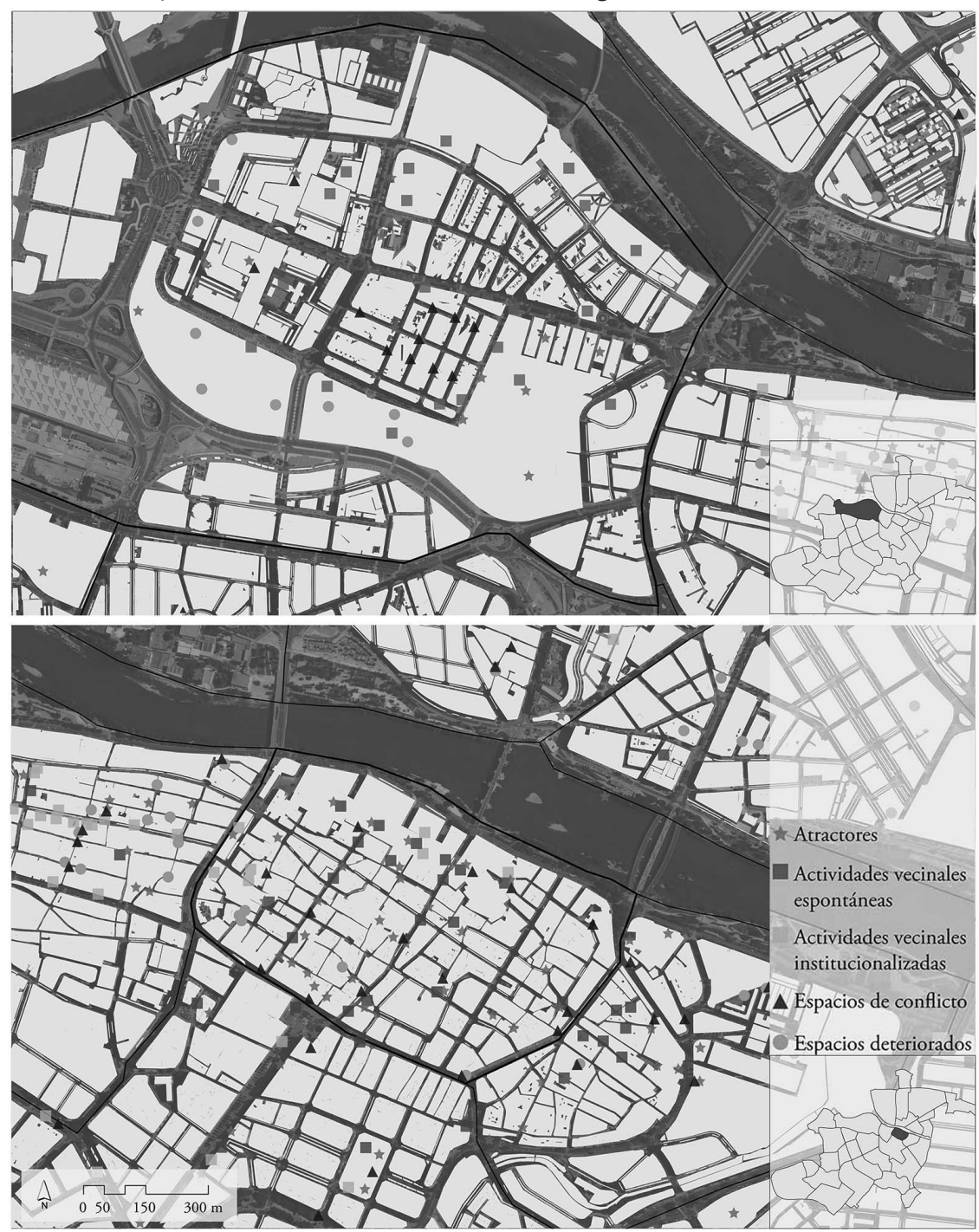

FUENTE ELABORACIÓN PROPIA, 2017 
El enfoque metodológico queda complementado por el diseño y realización de una encuesta desde una perspectiva cualitativo-estructural, formada por 36 preguntas -de respuesta tanto cerrada como, en determinados casos, abierta-, agrupadas de manera temática y orientadas a obtener información precisa acerca de la caracterización del barrio con respecto al conjunto de la ciudad, y ello en torno a tres aspectos básicos: sentimiento de pertenencia al barrio, intensidad de uso del espacio urbano e intensidad de relaciones sociales. El trabajo de campo se realizó durante los meses de junio de 2015 y noviembre de 2016. Una exhaustiva explicación sobre el diseño de la encuesta se puede encontrar en Cámara y Sorando (2017). La generación de este tipo de información permite establecer una gradación de los barrios según cuáles de sus espacios públicos, centros de asistencia primaria o locales de pequeño comercio son mejor valorados, y qué ámbitos de la ciudad y del barrio no alcanzan a proporcionar un servicio suficiente, según los propios vecinos. En otras palabras, este análisis de los hábitos y acciones de cotidianeidad por parte de los habitantes de los barrios permitiría comparar la distribución real de los elementos que caracterizan el barrio con respecto a los presentes en el imaginario colectivo. La información generada relativa a la percepción subjetiva de estos tres aspectos básicos parte del conocimiento local y se concreta en la definición de las siguientes variables: sentimiento de pertenencia al barrio (grado de vinculación con el barrio; deseo de cambiar de barrio), intensidad de uso del espacio urbano (adecuación del espacio libre y zonas verdes; adecuación de servicios y equipo urbano; agenda cultural, ocio y comercio) e intensidad de relaciones sociales (pertenencia a asociaciones; percepción de seguridad; percepción de riesgo de exclusión social). El análisis exploratorio de los datos generados para cada una de las ocho variables a través de la realización de las encuestas responde a la variabilidad de la distribución representada en la figura 5 .

\section{Resultados y discusión}

El análisis espacial demuestra que no existe una relación unívoca entre la presencia y distribución de atractores, por un lado, y la condición de vulnerabilidad de los barrios, por otro (figura 6). En realidad, se comprueba que los atractores refieren a plazas, equipamientos y espacios vinculados al tránsito peatonal, como calles peatonales -ligadas en la mayoría de los casos al comercio- o paseos. El estudio de los espacios materialmente deteriorados da soporte a esta perspectiva, ya que su distribución presenta una tendencia homogénea, independientemente de la condición de vulnerabilidad, y únicamente intensificada en determinadas áreas, revelando ciertos problemas de diseño en los bordes urbanos de la ciudad. La presencia de espacios de conflicto tampoco responde a la condición de vulnerabilidad de un barrio, dado que su distribución es mayoritariamente homogénea para todos los barrios estudiados, si bien su concentración aumenta en torno a determinados espacios urbanos. Incluso, es reseńable que exista un mayor número de ellos en ciertos barrios (como La Almozara) que no son valorados como problemáticos en términos de vulnerabilidad, frente a otros que sí quedan caracterizados como tales. 
FIGURA 5 | Dispersión de las variables que caracterizan la percepción del espacio urbano por parte de los vecinos a nivel de barrio
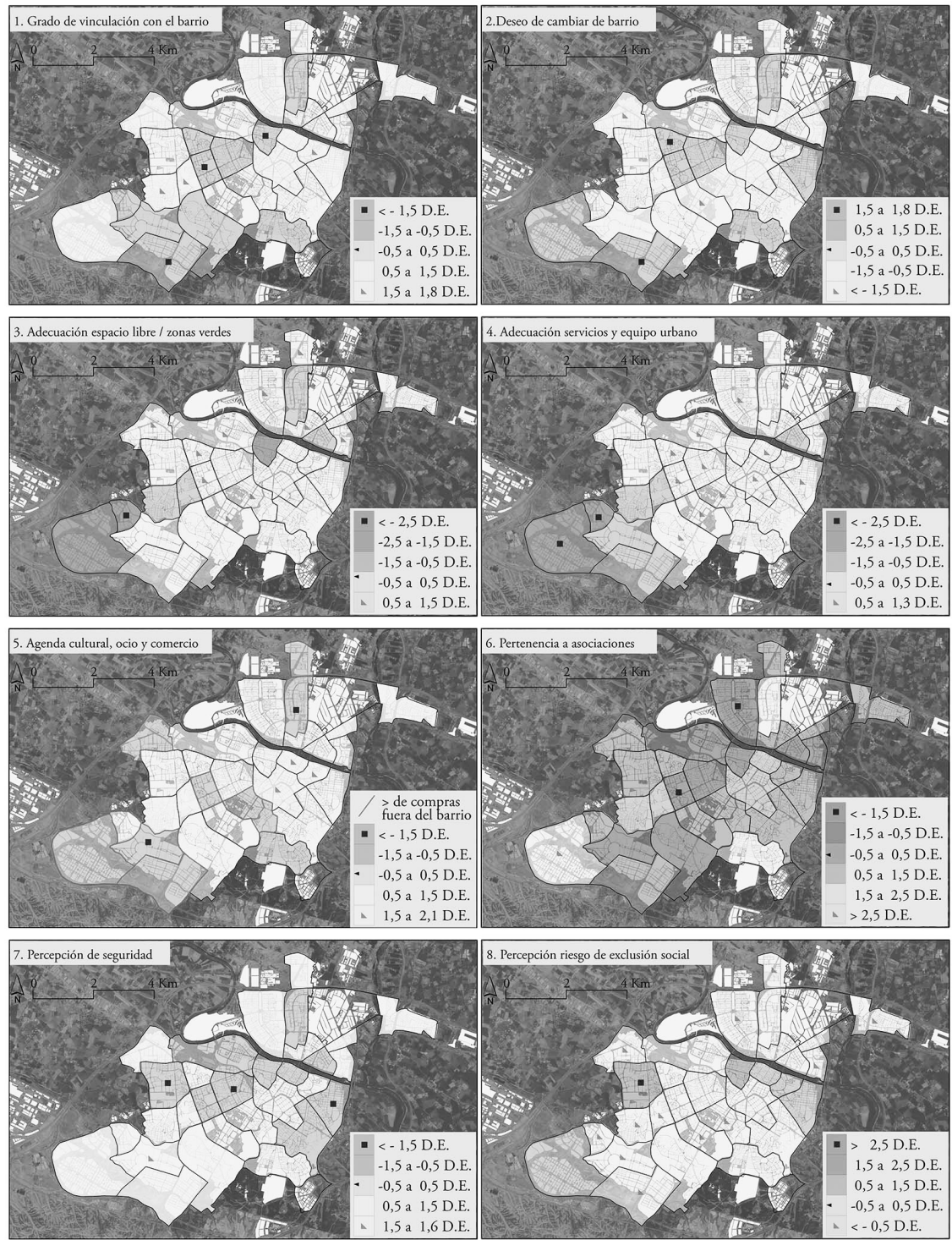

FUENTE ELABORACIÓN PROPIA, 2017

De acuerdo con los resultados, muchos de los barrios considerados vulnerables por las metodologías empleadas en los actuales informes de vulnerabilidad no se corresponden con aquellos en los que se ha detectado mayor número de espacios de riesgo y conflicto social. Es interesante seńalar que existe un predominio de plantas bajas con uso residencial, de terciario vacío o de solares en aquellos sectores de barrios que 
son considerados vulnerables. Del mismo modo, se demuestra que barrios en los que existe una mayor concentración de áreas de conflicto social no están caracterizados como vulnerables. Por lo tanto, tampoco puede concluirse que existe una relación unívoca entre los espacios de conflicto identificados, con respecto a la situación de vulnerabilidad o no vulnerabilidad de los barrios. Estos resultados apoyan la tesis de que las variables estadísticas demográficas, socioeconómicas y residenciales no son capaces por sí mismas de explicar ni categorizar el uso social del espacio público de un determinado barrio.

FIgURa 6 | Patrón de distribución de atractores, actividades vecinales y espacios de conflicto con respecto a las actuales áreas vulnerables en Zaragoza

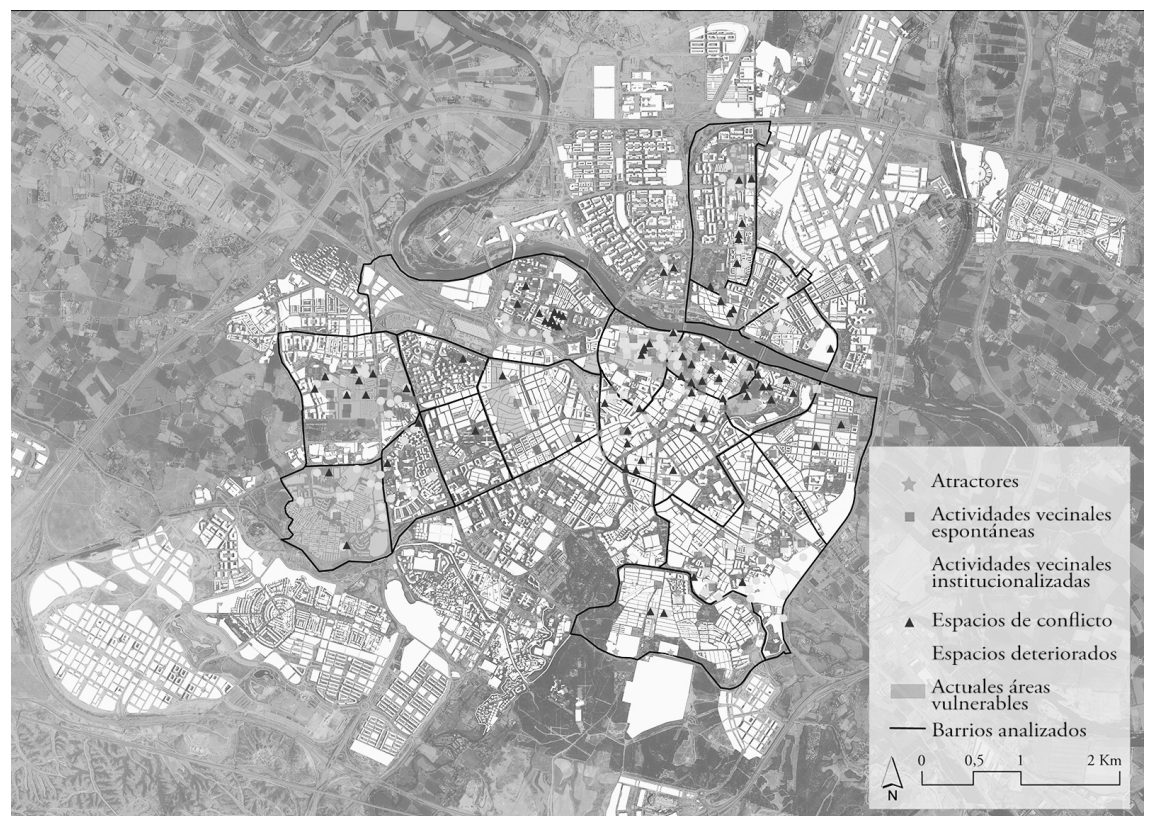

FUENTE ELABORACIÓN PROPIA, 2017

Sin embargo, sí se identifica presencia de actividades vecinales en aquellas áreas consideradas como vulnerables, de manera especial aquellas que surgen de modo espontáneo por parte de los vecinos y no tienen carácter institucional. Cabe señalar que esta proporción no se mantiene para el barrio de San Pablo (en continuidad con los barrios de La Almozara y Casco Histórico), donde existe una mayor presencia de actividades vecinales institucionalizadas que espontáneas. Los análisis espaciales indican que no existe una disminución de relaciones de cotidianeidad o ausencia de redes de apoyo en los barrios vulnerables frente a los no vulnerables, sino que el escenario es más complejo. En realidad, barrios no identificados como vulnerables por el Observatorio muestran unas redes de cotidianeidad intensas, al igual que otros sí identificados como vulnerables. Del mismo modo, determinadas áreas vulnerables de barrios como Delicias (al sur de La Almozara) o Picarral y Arrabal 
(ambos al otro margen del río Ebro) muestran una existencia débil o minoritaria de dichas relaciones de cotidianeidad. De acuerdo con estos resultados, los barrios vulnerables no son siempre los más conflictivos en términos sociales, ni se corresponden con los que presentan mayor deterioro físico.

Por su parte, el análisis exploratorio de datos sobre la percepción social a nivel de barrio viene a reforzar, de manera complementaria, estos resultados.

En primer lugar, e independientemente de la condición de vulnerabilidad, existe una tendencia generalizada por parte de los vecinos a no querer cambiar de barrio, cuestión que queda alineada con el grado de vinculación con el barrio. Los valores que más se alejan de la media, y que corresponden a aquellos barrios en los que el sentimiento de pertenencia es menor, refieren a los barrios de la zona sur, que han sido planificados y desarrollados en décadas más recientes, así como a los barrios, considerados vulnerables, de Delicias y San Pablo. Sin embargo, existen otros barrios vulnerables que presentan valores máximos de sentimiento de pertenencia; además, se constata que son estos barrios precisamente los que mayores valores muestran con respecto al conjunto de la ciudad. A tenor de los resultados, no puede demostrarse que existe una relación unívoca entre el grado de vinculación y la situación de vulnerabilidad o no vulnerabilidad de los barrios.

En segundo lugar, la intensidad en el uso del espacio urbano demuestra que, según los vecinos, no todos los barrios de la ciudad presentan niveles adecuados de equipo urbano, servicios públicos y espacios libres. Los valores más desfavorables corresponden a aquellos barrios más periféricos del margen derecho de la ciudad, si bien el barrio de San Pablo alcanza asimismo valores altos. Conviene destacar que no existe correspondencia entre la frecuencia de compras diarias en el propio barrio, y de ocio y actividades culturales, con respecto a la condición de vulnerabilidad del barrio.

En tercer y último lugar, la medición de la intensidad de relaciones sociales a partir del grado de asociacionismo y la percepción de seguridad refleja los resultados que a continuación se exponen.

En términos generales, el tejido asociativo para el conjunto de los barrios alcanza valores deficientes, siendo menor del $40 \%$ la población a nivel de ciudad que pertenece a alguna asociación. A nivel de barrio no existe relación unívoca entre los niveles más bajos o más altos de asociacionismo y la condición de vulnerabilidad. Sin embargo, los resultados obtenidos a esta escala revelan una cuestión fundamental, dado que la pertenencia a asociaciones no garantiza cierta intensidad de actividades vecinales e institucionales. En realidad, no se demuestra que estos valores estén relacionados con el patrón de distribución e intensidad de las actividades vecinales. Existen ejemplos en los que un nivel alto de asociacionismo se corresponde a una existencia débil de redes de cotidianeidad (como Picarral o Torrero; este último ubicado en el sector sureste de la ciudad), de tal forma que se considera que los niveles mínimos de convivencia quedan complementados por la existencia de agrupaciones que, representando intereses colectivos, se encargan precisamente de intensificar tales redes de apoyo y convivencia.

Existen, asimismo, ejemplos de otros barrios donde los niveles altos de asociacionismo parecen responder a una determinada demanda de mejora de los servicios públicos y equipo urbano del lugar, escenario en el que el asociacionismo alcanza los 
valores más altos a escala de ciudad. A este respecto convendría profundizar en un futuro sobre la naturaleza de las asociaciones y el efecto que ellas tienen en el barrio. La variable referida a la percepción de seguridad por parte del imaginario colectivo manifiesta que tal percepción es adecuada para el conjunto de la ciudad. A pesar de que no todos los barrios considerados vulnerables obtienen valores bajos de percepción de seguridad, en este caso los valores más bajos en este campo corresponden a barrios vulnerables. No obstante, la variable explicativa refiere a la presencia de atractores, ya que la percepción de seguridad alcanza los valores mínimos para aquellos ámbitos en los que los atractores presentan una distribución más dispersa y menos concentración en torno a elementos urbanos específicos. En este sentido, la posibilidad de contar con esta información georreferenciada es clave a la hora de investigar si existe, por ejemplo, cierta correspondencia entre la presencia de determinados usos en planta baja y la percepción social de seguridad en el espacio público.

Teniendo en cuenta la incorporación de estas nuevas variables generadas a partir de la metodología de análisis social y cartografía de las relaciones sociales que se producen en el entorno urbanizado, aplicada al estudio de la vulnerabilidad urbana y exclusión social, es importante observar que los patrones de distribución de usos en planta baja, la red de atractores y de espacios de conflicto, los espacios materialmente deteriorados, la presencia de actividades vecinales o de carácter institucional, junto con la percepción de pertenencia, de adecuación y uso del espacio urbano, así como la intensidad de relaciones sociales generadas en él, contribuyen a la caracterización del barrio desde el punto de vista social. Sin embargo, la actual caracterización de la condición de vulnerabilidad a nivel de barrio no presenta una relación significativa con la existencia de redes de apoyo social.

\section{Conclusiones finales}

La localización y puesta en medida de la red de convivencia y apoyo social ha demostrado que, en términos de vulnerabilidad, las actuales metodologías aplicadas en el ejercicio de la planificación deben ser evaluadas y mejoradas para adaptarse a una realidad social en gran medida desconocida. Se ha comprobado que el supuesto de partida, por el que se consideraba que un barrio caracterizado como vulnerable no cuenta con una red de apoyo social, no se cumple. Se ha demostrado, así, que el riesgo de aislamiento social no es mayor en las áreas vulnerables identificadas por las anteriores metodologías de evaluación de riesgo y vulnerabilidad urbana. En este sentido, se ha cartografiado las relaciones de cotidianeidad, con objeto de que los resultados del análisis espacial realizado puedan ser instrumentalizados para incorporar con garantías el ámbito de lo social en el desarrollo y diseño de la ciudad. La metodología empleada permite inferir los riesgos potenciales que afectan a la convivencia a nivel de barrio, por lo que adquiere un carácter necesario y complementario (no sustitutivo) a las actuales metodologías, además de considerarse como un documento valioso de carácter informativo a los ciudadanos, a sumar a los actuales documentos vigentes de planificación y gestión de la ciudad. La metodología de análisis empleada permite obtener información geoespacial para la caracterización de los 
conceptos de vulnerabilidad y exclusión social, hasta ahora fundados de manera esencial en el carácter físico y morfológico de la ciudad.

Los resultados son específicos de la ciudad de Zaragoza y no se pueden generalizar a otras ciudades o ámbitos urbanizados. Debido a que las redes de convivencia se construyen socialmente, su importancia se sitúa en la cartografía de un tiempo y lugar concretos. Sin embargo, aquí se ha puesto de manifiesto la validez del diseño metodológico para el ejercicio de la regeneración urbana y de desarrollo urbano integrado (Comisión Europea, 2014), así como la posibilidad de exportarlo a otros casos de estudio. Asimismo, se revela la importancia de la generación de fuentes primarias en términos de sostenibilidad social y de la sistematización de la información realizada, hasta ahora inexistente. Este trabajo sirve como punto de partida en la elaboración de otros estudios no solo de mayor especificidad (potencial de uso comercial, concentración y diversidad de usos, percepción de seguridad y vinculación con usos en planta baja), sino diseñados para abarcar un periodo temporal que permita comparar cómo ha variado la conformación de esta red de apoyo y relaciones sociales de cotidianeidad.

Los resultados que se obtienen aplicando esta metodología de análisis social del espacio público evidencian la importancia de descubrir cómo funciona el espacio urbano desde un punto de vista social, y cuál es su correspondencia con las variables que actualmente se consideran explicativas de la vulnerabilidad urbana. Esta perspectiva refuerza una posible vía de estudio que profundice en el estudio de cómo influyen las asociaciones de barrio, según su naturaleza, en la condición de vulnerable y de soporte de redes de apoyo a nivel de barrio en la ciudad. Este escenario más complejo revela, además, que la cartografía de los atractores y, sobre todo, de los espacios de conflicto refiere a una escala de mayor detalle que la generada -más genérica- por el conjunto de las metodologías de análisis de la vulnerabilidad urbana hasta ahora conocidas. En efecto, mientras que todas ellas toman la sección censal como área mínima a la que se atribuye una determinada condición de vulnerabilidad, el trabajo aquí presentado identifica la relación existente entre la presencia de atractores y espacios de conflicto, con respecto a los usos, elementos urbanos y estructura del espacio urbano. La diferencia es esencial, ya que trata de analizar un espacio vivido frente a un espacio delimitado.

\section{Agradecimientos}

Este artículo recoge resultados de la investigación "Mapa de Riesgo Social”, financiada por el Ministerio de Economía y Competitividad, Programa de $\mathrm{I}+\mathrm{D}+\mathrm{i}$ y orientada a los Retos de la Sociedad, 2013. Ref. CSO2013-42576-R. 


\section{Referencias bibliográficas}

Alguacil, J. (2006). Barrios desfavorecidos: Diagnóstico de la situación española. En F. Vidal Fernández (ed.), V Informe fuhem de politicas sociales: la exclusión social y el estado del bienestar en España (pp. 155-168). Madrid: Fundación Benéfico-Social Hogar del Empleado (FUHEM).

Alguacil, J. (2011). Cómo se hace un trabajo de investigación en sociología. Madrid: La Catarata. Álvarez, A. \& Roch, F. (2010). Regeneración urbana integrada en Europa. Documento sintesis. Valladolid: Instituto Universitario de Urbanística, Universidad de Valladolid.

Appleyard, D. \& Lintell, M. (1972). The environmental quality of city streets: The residents' viewpoint. Journal of the American Institute of Planners, 38(2), 84-101. https://doi. org/10.1080/01944367208977410

Arias, F. (coord.). (2000). La desigualdad urbana en España. Madrid: Ministerio de Fomento, Dirección General de Programación Económica y Presupuestaria, Centro de Publicaciones. http://habitat.aq.upm.es/due/afari.html

Atkinson, A. B. \& Kintrea, K. (2001). Disentangling area effects: Evidence from deprived and non-deprived neighbourhoods. Urban Studies, 38(12), 2.277-2.298. https://doi. org/10.1080/00420980120087162

Atkinson, A. B., Cantillon, B., Marlier, E. \& Nolan, B. (2002). Social indicators. The EU and social inclusion. Nueva York: Oxford University Press.

Bruquetas, M., Moreno, F.-J. \& Walliser, A. (2005). La regeneración de barrios desfavorecidos. Madrid: Fundación Alternativas. http://www.fundacionalternativas.org/laboratorio/ documentos, documento de trabajo 67.

Cámara, C., León, J. \& Ruiz, A. (2016). Mapa de riesgo social de Zaragoza. De técnica de gestión de poblaciones a praxis de consolidación de los comunes. En L. Diego, J. Fernández-Santos \& J. León (eds.), Open sourcing. Investigación y formación avanzada en arquitectura (pp. 77-103). Zaragoza: Universidad San Jorge.

Cámara, C. \& Sorando, D. (2017). Valoración de las percepciones subjetivas de los barrios como complemento al cálculo cuantitativo de riesgo de exclusión social. El caso del MrSz en Zaragoza. Clivatge. Estudios y testimonios sobre el conflicto y el cambio social, (5), 240-289. http://revistes.ub.edu/index.php/clivatge/article/view/18610/21117

Campbell, E., Henly, J. R., Elliott, D. S. \& Irwin, K. (2009). Journal of Urban Affairs, 31(4), 461-490. https://doi.org/10.1111/j.1467-9906.2009.00450.x

Comisión Europea. (2014). Desarrollo urbano sostenible integrado. Política de Cohesión 20142020. https://bit.ly/2CsMeAw

Conway, M. \& Konvitz, J. (2000). Meeting the challenge of distressed urban areas. Urban Studies, 37(4), 749-774. https://doi.org/10.1080/00420980050004008

Coulton, C. J., Korbin, J., Chan, T. \& Su, M. (2001). Mapping residents' perceptions of neighborhood boundaries: A methodological note. American Journal of Community Psychology, 29(2), 371-383. https://doi.org/10.1023/A:1010303419034

Cutter, S. L., Boruff, B. J. \& Shirley, W. L. (2003). Social vulnerability to environmental hazards. Social Science Quarterly, 84(2), 242-261. https://doi.org/10.1111/15406237.8402002

Cooley, Ch. H. (1909). Social organization: a study of the larger mind. Nueva York: Charles Scribner's Sons. 
De Miguel, A., Díez, J. \& Medina, A. (eds.). (1967). Tres estudios para un sistema de indicadores sociales. Madrid: Fundación Foessa.

De Miguel, A. (1970). Indicadores e indices en Sociología. Boletín de documentación del fondo para la investigación económica y social, Madrid, Confederación Espańola de Cajas de Ahorros, Vol. II, Fascículo 4.

Elliot, D. S., Wilson, W. J., Huizinga, D., Sampson, R. J., Elliott, A. \& Rankin, B. (1996). The effects of neighborhood disadvantage on adolescent development. Journal of Research in Crime and Delinquency, 33(4), 389-426. https://doi. org/10.1177/0022427896033004002

García-Bueno, G. (2017). Capital socio-urbanístico y potencial de regeneración urbana en Zaragoza. Clivatge. Estudios y testimonios sobre el conflicto y el cambio social, (5), 199239. http://revistes.ub.edu/index.php/clivatge/article/view/18609

Gehl, J. (2010). Cities for people. Washington, D.c.: Island Press.

Goicoechea, M. E. (2014). El mapa social de Buenos Aires (2001). En López-Roldan \& Fachelli, Técnicas multivariadas aplicadas al análisis de la realidad social. Seminario de Doctorado 2013, Facultad de Ciencias Sociales, Universidad de Buenos Aires. https:// ddd.uab.cat/pub/trerecpro/2014/117077/TFG_megoicoetxea.pdf

Guest, A. M. \& Lee, B. A. (1984). How urbanites define their neighborhoods. Population and Environment, 7(1), 32-56. https://doi.org/10.1007/BF01257471

Haklay, M. (2013) Neogeography and the delusion of democratization. Environment and Planning A, (45), 55-69. https://doi.org/10.1068/a45184

Hernández-Aja, A., Vázquez-Espí, M., García-Madruga, C., Matesanz-Parellada, Á., MorenoGarcía, E., Alguacil-Gómez, J. \& Camacho-Gutiérrez, J. (2013). Análisis urbanistico de barrios vulnerables. Madrid: Observatorio de Vulnerabilidad Urbana de España. http://habitat.aq.upm.es/bbvv

Keestelot, C. (2008). Urban socio-spatial configurations and the future of European cities. En Y. Kazepov (ed.), Cities of Europe: Changing contexts, local arrangements, and the challenge to urban cohesion (pp. 123-148). Oxford: Blackwell Publishing.

Keller, S. (1968). The urban neighborhood: A sociological perspective. Nueva York: Random House.

King, G., Keohane, R. \& Verba, S. (2000). El diseño de la investigación social. La inferencia cientifica en los estudios cualitativos. Madrid: Alianza Editorial.

Kingston, B., Huizinga D. \& Elliot, D. S. (2009). A test of social disorganization theory in high-risk urban neighborhoods. Youth \& Society, 41(1), 53-79. https://doi. org/10.1177/0044118X09338343

Lee, Y. J. (2014). Social vulnerability indicators as a sustainable planning tool. Environmental Impact Assessment Review, (44), 31-42. https://doi.org/10.1016/j.eiar.2013.08.002

León, J. \& García-Carpintero, G. (2017). Mapa de riesgo social de Zaragoza. Una visión alternativa a los análisis urbanísticos de vulnerabilidad urbana. Clivatge, (5), 11-47. http://revistes.ub.edu/index.php/clivatge/article/view/18603

Lofland, J. \& Lofland, L. H. (1995). Analyzing social settings: a guide to qualitative observation and analysis. Belmont, CA: Wadsworth.

Maclaren, V. W. (1996). Urban sustainability reporting. Journal of the American Planning Association, 62(2), 184-203. https://doi.org/10.1080/01944369608975684 
Ministerio de Fomento, España (1990). Análisis urbanistico de Barrios Vulnerables en España. Madrid: Gobierno de España.

Murie, A. \& Musterd, S. (2004). Social exclusion and opportunity structures in European cities and neighbourhoods. Urban Studies, 41(8), 1.441-1.459. https://doi. org/10.1080/0042098042000226948

Musterd, S. \& Murie, A. (eds.) (1999). The spatial dimension of urban social exclusion and Integration. Final report. Urbex series, 22. Amsterdam: University of Amsterdam.

Obst, P. L. \& White, K. M. (2004). Revisiting the Sense of Community Index: A confirmatory factor analysis. Journal of Community Psychology, 32(6), 691-705. https://doi. org/10.1002/jcop.20027

Organisation for Economic Co-operation and Development (OECD). (1998). Integrating distressed urban areas. París: OECD Publishing. https://doi.org/10.1787/9789264162884-en

Rana, S. \& Joliveau, T. (2009) NeoGeography: an extension of mainstream geography for everyone made by everyone? Journal of Location Based Services, (3), 75-81. https://doi. org/10.1787/9789264162884-en 10.1080/17489720903146824

Raya, E. (2007). Exclusión social: indicadores para su estudio y aplicación para el trabajo social. Revista del Ministerio de Trabajo e Inmigración, (70), 155-172. https://dialnet.unirioja. es/servlet/articulo?codigo $=2507760$

Raya, E. (2010) Aplicaciones de una herramienta para el diagnóstico y la investigación en exclusión social, Documentos de Trabajo Social, (48), 117-136. http://www. trabajosocialmalaga.org/archivos/revista_dts/48_06.pdf

Ruiz, A. \& Alfaro, P. (2017). Áreas de rehabilitación en la ciudad de Zaragoza: noción, encaje urbanístico y criterios de selección. Clivatge. Estudios y testimonios sobre el conflicto y el cambio social, (5), 170-198. http://revistes.ub.edu/index.php/clivatge/article/ view/18608/21115

Ruiz-Varona, A. \& León-Casero, J. (2017). Social risk map. The design of a complementary methodology to vulnerability indexes applied to urban rehabilitation activity. En 24th ISUF [International Seminar on Urban Form] International Conference 2017: City and Territory in the Globalization Age (pp. 157-166). https://doi.org/10.4995/ ISUF2017.2017.5060

Temes, R. (2014). Valoración de la vulnerabilidad integral en las áreas residenciales de Madrid. Eure, revista latinoamericana de estudios urbano regionales, 40(119), 119-149. http:// www.scielo.cl/pdf/eure/v40n119/art06.pdf

Torres, H. (1993). El mapa social de Buenos Aires 1940-1990. Buenos Aires: Facultad de Arquitectura, Diseño y Urbanismo, Universidad de Buenos Aires.

Turcu, C. (2012). Re-thinking sustainability indicators: local perspectives of urban sustainability. Journal of Environmental Planning and Management, 56(3), 695-719. https://doi.org/1 0.1080/09640568.2012.698984 